\title{
USING MULTI-CRITERIA MODELING AND SIMULATION TO ACHIEVE LEAN GOALS
}

\author{
Gerald W. Evans \\ Suraj M. Alexander \\ Department of Industrial Engineering \\ University of Louisville \\ Louisville, KY 40292, U.S.A.
}

\begin{abstract}
Lean principles require the identification of an ideal system state along with an associated policy to achieve that state. This paper discusses the use of multi-criteria models in conjunction with optimization procedures and simulation in order to identify an ideal system state and associated policy. An illustration involving the determination of a replenishment policy for a distribution system is described.
\end{abstract}

\section{INTRODUCTION}

The essence of lean manufacturing can be stated succinctly as the process of delivering value to the customer efficiently. This process is defined by the "value stream". The goal of lean manufacturing, therefore, is to eliminate waste in the value stream.

Lean manufacturing begins with defining customer needs and "optimizing" the process of satisfying these needs. In the manufacturing context, this would mean providing customers with high quality products that meet or exceed their requirements, in the right quantities, at the right time, at the lowest cost. The difficulty with implementing "lean" in manufacturing results from the fact that many of the control factors, that drive the value derived by customers, are often conflicting. For example, larger inventory or work in process (WIP), may enable providing customers with the right quantity of products at the right time, however, this may conflict with the objectives of lower cost and efficiency; smaller lot sizes in production and shipping may reduce inventory costs, but increase manufacturing and shipping costs; uncertainties related to equipment availability owing to break downs and scheduled maintenance, variations in customer requirements, quality deficiencies etc., may require the addition of inventory, or capacity, however, this would imply increased cost. Simulation enables the understanding of the implications of alternative solutions on multiple ob- jectives, thereby facilitating the selection of effective approaches towards lean.

One of the important steps in the lean manufacturing process involves defining the current state of the system and then an ideal state that can be pursued over time. These system states should be defined through the use of a set of valid performance measures (also called attributes). Examples of such performance measures would be work in process, production rates, number of defective items produced, percentage of deliveries made on time, number of lost sales, etc.

Defining an ideal state then just means specification of the desired values for the performance measures. However, it may not be possible to attain the desired values for all of the performance measures, since typically tradeoffs must be made between various pairs of performance measures. For example, it may not be possible to attain simultaneously the ideal values for both lost sales (which would result when inventory levels for specified items are zero) and inventory holding costs.

One way to specify the tradeoffs of a decision maker is through the use of a criterion model. The next section of this paper discusses various examples of criterion models used in simulation studies, while the third section presents some principles from the area of multi-objective decision analysis that can be used in formulation of criterion models. The fourth section presents a case study involving the determination of an operating policy for a distribution system. Finally, the last section of the paper provides a summary.

\section{DEFINITION AND EXAMPLES OF CRITERION MODELS USED IN SIMULATION}

A criterion model is just a way of combining all of the outputs from the simulation model so that a ranking of the various alternatives associated with the simulation study can be accomplished. More formally, given any two sets of outputs (each associated with respective alternatives) 


\section{Evans and Alexander}

from experimentation with a simulation model, the decision maker can say that Output Set 1 is preferred to Output Set 2, Output Set 2 is preferred to Output Set 1, or indifference exists between the two sets of outputs, through the use of a criterion model.

The development and eventual choice of a criterion model is an important one, as ultimately this choice will affect the alternative chosen for implementation. In addition, this choice will (or at least should) affect the design of the simulation model itself, the set up for the experimental runs of the simulation, and the choice of any optimization procedure used in the study. For example, the simulation model must be designed in such a way that the performance measure values required by the criterion model are output by the simulation model.

Of course the major determining factor for the choice of a criterion model is that it accurately represents the preference structure of the decision maker(s) (just as the simulation model should be an accurate representation of the real system). The key phrase here is: "an accurate representation"- the more effort that is placed into developing the criterion model, the more accurate it will be, and the more likely it is that the choice of a correct alternative will be made as a result. Hence, as is typically the case in the development of any type of model, there is a tradeoff between effort and accuracy in the development of a criterion model. One wants the criterion model to be accurate enough so that the "best" alternative is selected for implementation.

Two major considerations must be accounted for in the development and use of a criterion model in conjunction with a simulation study. The first consideration is just what should the underlying criterion model be. This implies the choice of performance measures (or attributes), as well as how these measures are to be combined to allow for the inherent tradeoffs between the various pairs of measures. The second consideration, more subtle in nature than the first, is how to address the fact that typically one can only achieve estimates of important performance measure values through experimentation with a simulation model. This second consideration has important implications for procedures to be used in experimentation and optimization.

One of the characteristics of most simulation models is the large number, and wide variety, of performance measures which they can output. Indeed, since a simulation model typically "recreates" the processes associated with a system, performance measure values are computed by executing the model and collecting output data, as opposed to just computing performance values as closedform function of input/decision variables. In addition, although often glossed over, what one obtains in collecting output data from a simulation model are estimates of performance measure values, not the actual values. This fact can have important implications for both the criterion model chosen and the set up for experimentation used for the simulation analysis.

There are a wide variety of criterion models that are used in conjunction with simulation models described in the literature. April et al (2004) describe several examples in their paper for various application areas involving simulation, as described below. For example, in a production problem for which the alternatives are described in terms of various sets of machine capacities, they suggest the following criterion model:

$$
\begin{aligned}
& \text { Maximize Factory Throughput } \\
& \text { Subject to: Work-in -Process } \leq \mathrm{B}_{1} \text {, and } \\
& \text { Cost } \leq \mathrm{B}_{2}, \\
& \text { where } \mathrm{B}_{1} \text { and } \mathrm{B}_{2} \text { are constants. }
\end{aligned}
$$

Note that in this example, the performance measures are Factory Throughput, Work-In-Process Inventory, and Cost, and that there are many different criterion models that one could form with these performance measures; for example, one could use a criterion model involving the minimization of work-in-process inventory, subject to constraints on factory throughput and cost. Note also that this criterion model does not address the stochastic nature of these performance measures - that is, for any particular alternative, the values for the three performance measures can be represented as random variables.

In a problem involving project portfolio optimization, the alternatives can be described in terms of which projects will be selected for investment by a company. April et al (2004) suggest several different criterion models for this situation, including the following:

\section{Maximize E (NPV) \\ Subject to: SD (NPV) $\leq \$ 140,000,000$., and a Budget Constraint.}

In this criterion model, the two major performance measures are the Net Present Value associated with the income from the investments, and the cost associated with the investments (i.e., the budget). Note that the uncertainty in the estimate of the NPV is accounted for by placing a constraint on the standard deviation (SD) of the estimate of NPV. April et al (2004) provide an alternative approach to consider this uncertainty with the following criterion model:

$$
\begin{aligned}
& \text { Maximize E }(\mathrm{NPV}) \\
& \text { Subject to: } \mathrm{PR}(\mathrm{NPV} \leq \$ 176,000,000)<=.05 \text {., } \\
& \text { and a Budget Constraint. }
\end{aligned}
$$




\section{Evans and Alexander}

In this model, the first constraint can be stated in words as "the probability that the net present value is less than or equal to $\$ 176,000,000$ should be less than or equal to .05 ". At first glance, this would seem to be a reasonable criterion model; but, note how this criterion model would favor an outcome stated as "achieving a net present value of $\$ 177,000,000$, with probability of .96 and a net present value of $\$ 100,000,000$ with a probability of .04 " over an outcome stated as "achieving a net present value of $\$ 175,000,000$ with a probability of .06 and a net present value of $\$ 200,000,000$ with a probability of $.94 ”$. The second outcome would not even be considered since it is infeasible, yet it would be difficult to imagine a decision maker who would prefer the first outcome over the second.

Finally, in the area of project portfolio optimization, April et al (2004) present the criterion model given by:

$$
\begin{aligned}
& \text { Maximize Probability (NPV } \geq \$ 394,000,000) \\
& \text { Subject to: } \begin{array}{l}
\text { Budget Constraints and } \\
\text { Personnel Constraints. }
\end{array}
\end{aligned}
$$

Each of the above criterion models, when optimized with the simulation model, could lead to the selection a different "optimal" alternative, and corresponding outcome. This then suggests one, ad hoc, approach to the determination of a criterion model: Try several different criterion models, present the resulting solutions and corresponding outcomes to the decision maker(s), and then have the decision maker(s) select the preferred outcome. The criterion model associated with this preferred outcome is the preferred one. Of course, such an approach assumes the existence of enough resources to perform the required analysis. It also assumes that, out of all of the criterion models developed, and out of the wide variety that could have been used, one of these is the best one to use.

In a paper involving supply chain management, Jain (2004) employed what might be termed a "scorecard approach" as a criterion model. In this approach, the decision maker(s) are presented with the output (as measured by several performance measures) for several alternatives, and then asked to select the preferred alternative. Hence, the criterion model is never made explicit, but instead is assumed to exist in the minds of the decision maker(s), allowing them to rank the alternatives associated with the outcomes, or to at least select the "best" outcome. Of course, this scorecard approach, involving the use of an implicit criterion model, is not appropriate when some type of automatic optimization procedure is needed. Such an automatic optimization procedure is needed when there are so many alternatives (as in, for example, when the alternatives are defined by a combination of decision vari- able values) that only a fraction of them can be explicitly evaluated.

In particular, Jain (2004) used a simulation model in order to study the impacts of six control parameters on three key performance measures: service levels, inventory investments, and order-to-delivery lead times, for a large logistics supply chain with customers located in 50 states and 27 countries. The fact that tradeoffs must be made among the three performance measures is obvious. In order to keep the analysis relatively simple, a key control variable, administrative business process time (ABPT) was employed. Using the simulation model, the control parameters (from the six previously mentioned) which had a significant impact on ABPT were identified. Following this, again using the simulation model, graphs were developed illustrating the relationships between ABPT (as the independent variable) and the three performance measures (as dependent variables) mentioned above.

Butler, Morrice, and Mullarkey (2001) employ the use of a criterion model involving the maximization of expected utility. Their problem involved the design of a signal quality survey, which is a large outdoor operation requiring five types of crews: a layout crew, a recording crew, a signal crew, a packing crew, and a transport crew. These projects take place over a large geographical area and require anywhere from 20 to 1000 people, and capital equipment valued in the tens of millions of dollars. Butler, Morrice, and Mullarkey defined four alternatives (i.e., configurations) for analysis. Each of the four alternatives was defined by resource levels on two dimensions: the number of source crews and the number of units of receiving equipment. The utility function used in the criterion model was defined as a function of ten performance measures: survey cost, survey duration, productivity for each of four crew types, and worker satisfaction for each of four crew types. Of the five crew types, only the recording crew was not included as one of the performance measures since this type of crew did not bottleneck production. Productivity and worker satisfaction were each defined as functions of utilization.

One of the difficult methodological aspects of Butler, Morrice, and Mullarkey's work involved the fact that only an estimate of expected utility (for any specific alternative configuration) could be obtained through experimentation with the simulation model. Therefore, one can never be absolutely positive that the alternative which maximizes expected utility is selected. Hence, the criterion model used might be stated as:

Maximize Expected Utility subject to: the constraint that the probability of correct selection is greater than or equal to $\mathrm{P}^{*}$, whenever the difference in the expected utility values for the best and second best alternatives is greater than or equal to $\delta^{*}$. 


\section{Evans and Alexander}

Correct selection means that the alternative which gives the largest expected utility is selected. $\mathrm{P}^{*}$ and $\delta^{*}$ must be input by the decision maker for this criterion model. $\mathrm{P}^{*}$ is typically set at a value of .9 or .95 .

One of the difficulties associated with the use of the above criterion model is that $\delta^{*}$ should have some inherent meaning to the decision maker. Since the difference in the expected utilities for two alternatives would have no inherent meaning to a decision maker, like, for example, the difference in expected daily production rate, or the difference in expected cost, the use of the above criterion model becomes problematic in nature.

Butler, Morrice, and Mullarkey (2001) suggest a twostep approach in the determination of $\delta^{*}$. The first step involves defining $\delta_{j}^{*}$ for each of the various individual attributes (performance measures) of the utility function. Since there are ten attributes in their problem, ten different values for "indifference amounts": $\delta_{1}{ }^{*}, \delta_{2}{ }^{*}, \ldots, \delta_{10}{ }^{*}$ must be provided by the decision maker. Then, in the second step, $\delta^{*}$ is defined as a weighted combination of these individual indifference amounts. See Butler, Morrice, and Mullarkey (2001) for additional details.

Anderson, Evans, and Biles (2006) applied simulation and criterion models to the design of a logistics system involving barge operations for the delivery of petroleum products. The criterion model used was a single attribute utility function with the attribute of total solution cost. This total solution cost was the sum of three different costs: penalty cost (resulting from backorders), variable transportation cost, and inventory holding costs. A scatter search algorithm was employed to perform the optimization.

Lee et al (2004) present an approach to ranking and selection in the presence of multiple objectives in which no explicit criterion model is used. Their paper addresses the problem of determining the number of replications required for each alternative in order to find the set of nondominated outcomes. A non-dominated outcome might be loosely defined as one in which each performance measure value is at least as good as the associated performance measure value for any other outcome associated with a feasible alternative, and better on at least one performance measure value. Once this set is determined, a decision maker, or a group of decision makers, could select a bestcompromise alternative through the use of an implicit criterion model. Such an approach would be especially useful when there are many decision makers involved in the process.

Finally, consider the optimization software package, OptQuest (Schwetman 2000), which is an optimization "add-in" for several simulation packages.. OptQuest implicitly employs a criterion model which can be described as maximizing or minimizing a performance measure value, subject to three types of constraints: first, constraints related to placing upper and lower bounds on control va- riable values; second, constraints on functions of control variables (e.g., the sum of capacities over all resources must be less than or equal to 10); and, third, constraints related to the placing of upper and or lower bounds on performance measure values. Note that the various alternatives are defined in this case in terms of the values assigned to the control variables. OptQuest allows two basic approaches to set the precision of the output. The user may just specify a particular number of replications for each alternative simulated, or the user may specify a particular half-width for the $95 \%$ confidence interval associated with whatever performance measure is being maximized or minimized. The system will make the required number of replications to achieve this precision. Note that the precision for the other performance measures (not considered in the objective function) are only considered in an implicit fashion.

\section{DETERMINATION OF A CRITERION MODEL}

As noted in the Introduction, there are two basic steps associated with the determination of a criterion model to be used in conjunction with a simulation model. The first step is the determination of the attributes (or performance measures) for the criterion model. The second step is the determination of how the attributes should be combined into a criterion model.

\subsection{Determination of Attributes}

Attributes basically refer to the important performance measures for making decisions about the system. The attributes could refer to expected values/mean values (e.g., mean flowtime for all patients processed in the emergency room, mean utilization of a machine, mean number of customers in a queue, etc.), proportions or fractions (e.g., the proportion of patients who wait longer than 1 hour for service in an emergency room, the proportion of arrivals to the drive-through in a fast food restaurant which balk because there are too many customers in line), maximum or minimum values for some quantity (i.e., the maximum number of parts in inventory over the run of the simulation model; the maximum amount of time, over all customers, that a restaurant patron has to wait for service) or others.

\subsubsection{Hierarchies of Objectives and Attributes}

Keeney (1992) notes that the attributes chosen for a value model (what we have termed a criterion model) should reflect the values of the decision maker(s) with respect to the system under study. The objectives and attributes for a decision problem typically form a hierarchy, with the major (or strategic) objectives towards the top of the hierarchy, and the tactical/operational objectives towards the 


\section{Evans and Alexander}

bottom of the hierarchy. At the very lowest level of the hierarchy should be the attributes for the system/decision problem. These attributes should provide a measure for how well the lowest level objectives (as well as the strategic objectives) are achieved. Examples of such hierarchies are given in Evans and Fairbairn (1989) for planning decisions at NASA, and Cambron and Evans (1991) for layout design problems.

Manheim and Hall (1967) suggest two related approaches that can be used in the development of a hierarchy from the top down: specification and means-end. In the specification approach, one specifies or categorizes the various ways of doing something. In the means-end approach, one relates the means by which something is achieved.

For example, consider a situation in which an analyst wants to develop a simulation model to be used in the development of a schedule for the personnel of a fast food restaurant. (See Karwhat (1991) and Love and Hoey (1990) for applications of OR/simulation in restaurants). A schedule might imply an assignment of each of the restaurant's employees to a particular set of time periods during the week, as well as a type of task to perform (e.g., order-pay and pick-up counter). The top level objective might be something that is typically strategic, yet nebulous in nature:

"Optimize the well-being of all persons asso ciated with the restaurant."

To expand on this initial, strategic, objective, the decision maker(s) would answer the question:

"What is the means by which we can opti mize the well being of all persons associated with the restaurant?"

The answers to this question would give us a set of lower level objectives, as follows:

"Optimize the well being of the restaurant's customers."

"Optimize the well being of the restaurant's employees."

"Optimize the well being of the restaurant's owners"

Note that these three objectives would also correspond to the "categorization" approach to specifying lower level objectives; i.e., the various categories of stakeholders (all persons associated with the restaurant) could be specified as customers, employees, and owners.

Now, in the development of the hierarchy, an analyst must remember to only include objectives and attributes which can be affected by the decision in question. For ex- ample, in the problem mentioned above, in which the decision has to do with the best schedule for the restaurant personnel, attributes related to maximizing the satisfaction of the restaurant's customers should not address the quality of the food, since the quality of the food will not be affected by the restaurant personnel schedule (accept possibly in some extreme sense). However, attributes related to customer waiting time/flow time could be (and probably should be) included, since these would be affected by the schedule chosen for restaurant personnel.

Returning to our hierarchy, consider the second objective at the second level of our hierarchy: optimize the well being of the restaurant's employees. The means by which this could be achieved could be stated in several different ways (keeping in mind that means must relate to the working schedule for the employee). For example, this means might be stated as any of the following:

1. Maximize the number of employees who get their first choice for a working schedule.

2. Maximize the number of employees who receive one of their first three choices for a schedule.

On the other hand, the decision maker might prefer to give more weight to employees with more seniority, as a reward for those employees.

Consider the third objective at the second level of the hierarchy: "optimize the well-being of the restaurant's customers". As with the objective relating to the restaurant's employees, one could think of several means by which this could be accomplished. For example, the following objectives might be stated:

1. Minimize the fraction of customers who must wait longer than 5 minutes to place their order.

2. Minimize the average amount of time that a customer must wait prior to placing his or her order.

3. Minimize the average amount of time that a customer must wait prior to receiving his or her order.

The last (or lowest) level of the hierarchy will consist of the attributes for the criterion model, typically one attribute for each lowest level objective. Normally, assuming the lowest level objectives are well defined, association of an attribute with an objective is fairly straightforward. For example, if the objective is to "minimize the fraction of customers who must wait longer than 5 minutes to place their order", then an obvious attribute is "the fraction of customers who must wait longer than five minutes to place their order". 


\subsubsection{Categorization of Attributes}

Attributes can be categorized along two distinct dimensions: natural or constructed, and proxy or nonproxy. That is any attribute will be one of four types: natural-proxy, natural-nonproxy, constructed-proxy, and constructednonproxy.

Natural attributes are typically quantitative in nature, and can normally be easily defined when the associated objective is well-defined. Examples would be attributes such as those mentioned above: "the fraction of customers who must wait longer than five minutes to place their order", or "the cost in dollars for a particular employee schedule". Also, when the hierarchy is fairly detailed in nature (i.e., expanded about as much as possible), then normally the attributes associated with the lowest level objectives will be natural attributes.

Constructed attributes (called subjective attributes by many authors) are typically associated with more nebulous objectives. These more nebulous objectives often correspond to those objectives that are strategic (called fundamental objectives by Keeney 1992) in nature, and are therefore found at a higher level in the hierarchy. One might also consider these as subjective, or qualitative, in nature, in that one associates a subjective scale with these attributes. Examples of such attributes would be: "the overall satisfaction of our employees with their working schedules, measured on a subjective scale of 1 to 5 , or "the efficiency of our production operation, measured on a scale of 1 to 5". One should usually associate a phrase with each number in a subjective scale for a constructed attribute. For example, with the constructed attribute: employee satisfaction above, one might associate the phrase: "extremely satisfied" with the number 5, and the phrase: "extremely dissatisfied" with the number 1.

The major difficulty with a constructed attribute is that its evaluation is subjective in nature. For example, for one qualified decision maker (e.g., an owner or manager of the restaurant) a set of weekly working schedules (one schedule for each employee) could correspond to a set for which he/she (the decision maker) would say the employees would be extremely satisfied with. For another, but equally qualified, decision maker, this set of schedules might translate into moderate satisfaction of the employees. See Keeney (1992) for additional discussion of constructed attributes.

Since outputs from a simulation model are quantitative in nature, any attributes computed directly from simulation model outputs will be natural attributes, not constructed attributes. Of course one could employ constructed attributes in conjunction with a simulation model by just having a decision maker analyze the output of a model for a specific alternative, and render a subjective evaluation with respect to a constructed attribute. This may be appropriate if there are many attributes required to measure the performance of a system, as in the case when a manufacturing system produces hundreds of different part types (Evans, Biles, and Alexander 1992). However, such an approach may preclude the use of any sophisticated optimization procedure in the search for an optimal solution.

A proxy attribute is one which represents an indirect measure for an associated objective (Keeney 1992). For example, the satisfaction of a restaurant employee with his or her working schedule might be indirectly measured by the percentage of time he or she arrives to work on time. As another example, a proxy attribute associated with optimizing the effectiveness of an ambulance service would be the average response time for the service. The advantage of a proxy attribute is that such an attribute is often more easily evaluated than a more direct measure for any specific objective. The disadvantage of using a proxy attribute is that, by definition, it is only an indirect measure of an objective (i.e., the relationship between the value of the proxy attribute and the achievement of the associated objective may not be known with certainty). See Keeney, page 103 (1992) for additional discussion concerning the use of a proxy attribute for a means objective (a lower level objective) vs. use of a proxy attribute for a fundamental objective.

Proxy attributes can be very useful in the construction of criterion models to be used with a simulation model. In the example noted above, measuring the satisfaction of a restaurant's customers could be accomplished with the proxy attribute: the fraction of customers who must wait longer than five minutes to place their order. The value for such an attribute could be easily computed by a simulation model. Of course, in order to prove useful, the decision maker must have an intimate knowledge of the relationship between a customer's waiting time and his or her satisfaction. In this case, the decision maker would have to know that most customers would have a threshold waiting time of five minutes. (Note how this threshold waiting time could change depending on the country in which the restaurant is located).

Finally, Keeney and Raiffa (1993) suggest that a set of attributes for a decision problem satisfy several characteristics, labeled as completeness, operability, decomposability, lack of redundancy, and minimum size.

\subsection{Combining the Attributes into a Criterion Model}

The key concept associated with combining of attributes into a criterion model has to do with representing the preference structure of the decision maker(s) in an accurate fashion. Hence, the criterion model must account for the tradeoffs among the various pairs of attributes as well as the uncertainty (and inherent risk) in the values for the attributes as estimated from the simulation model. 


\section{Evans and Alexander}

A general form for a criterion model can be stated as follows:

Maximize/Minimize $\mathrm{f}\left(\mathrm{X}_{1}, \mathrm{X}_{2}, \ldots, \mathrm{X}_{\mathrm{n}}\right) \mid \mathrm{g}_{\mathrm{i}}\left(\mathrm{X}_{1}, \mathrm{X}_{2}, \ldots\right.$, $\left.X_{n}\right) \leq, \geq$, or $=B_{i}$, for $i=1, \ldots, n$,

where $\mathrm{f}$ is the objective function, $\mathrm{g}_{\mathrm{i}}$ are constraint functions, $B_{i}$ are constants, and the $X_{1}, X_{2}, \ldots, X_{n}$ are the attributes of the problem.

Examples of objective functions include the following: expected value of a multi-attribute utility function, a multi-attribute value function (see Chapter 3 of Keeney and Raiffa 1993), an attribute value (i.e., $X_{i}$, for a particular $\mathrm{i}$ ), and the probability that the value for attribute $\mathrm{i}$ is greater than or equal to a particular number $\operatorname{Pr}\left(X_{i} \geq .9\right)$.

Examples of constraints include the following: the probability that $X_{\mathrm{i}}$ is greater than or equal some constant is less than or equal to a specific probability (e.g., the probability that the waiting time of a customer at our fast food restaurant is greater than or equal to 5 minutes should be less than or equal to .05), and the expected value of an attribute should be greater than or equal to some constant (e.g., the expected utilization of our average worker should be greater than or equal to .8 ).

One important factor to keep in mind, related to whether to consider an attribute in a criterion model through the use of a constraint or through the use of an objective function, is that once a constraint is satisfied, no increased value can be attained by the improvement of the attribute value associated with the constraint. This fact was illustrated through some of the examples discussed in Section 2 above. Hence, if an attribute is of particular importance, and there is no "natural constraint" with which it is associated, then it may be best to include it within an objective function.

A multi-attribute utility function, by definition, is the most accurate representation of a decision maker's preference structure (see Chapter 16 of Clemen 1996). That is, a utility function (which is specific for a decision maker) is defined by the fact that the expected value of the utility function for a probabilistic outcome is greater than the expected value for the utility function for a different probabilistic outcome if and only if the decision maker prefers the first probabilistic outcome to the second. The major difficulty with the use of a utility function has to do with its assessment, or determination, especially when the number of attributes is large (e.g., greater than three or four). One reason for this difficulty has to do with the fact that, in order to accomplish the assessment, the decision maker(s) must answer questions concerning rankings/tradeoffs over probability distributions over the outcome space. These questions can be very difficult to answer. See Clemen (1996) for additional discussion concerning the assessment of multi-attribute utility functions.
In some sense, however, a multi-attribute utility function represents a very natural component of a criterion model for use in a simulation study, since the output from a simulation model is probabilistic in nature. However, as noted by Butler, Morrice, and Mullarkey (2001) and as mentioned earlier, when using expected utility as a portion of a criterion model, one must be concerned with the fact that only an estimate of expected utility can be attained for any particular alternative. The precision of the estimate can be improved with more replications, but it still remains an estimate. One could use an approach such as that described by Butler, Morrice, and Mullarkey (2001) or one could employ a less sophisticated approach, involving a specification that the confidence interval for expected utility be of a certain width. In many cases, it may be appropriate to assess a utility function over only a few of the most important attributes, while restricting the values of the other attributes through the use of constraints. This would alleviate the problem of assessment when many attributes are involved.

\section{A CASE STUDY INVOLVING A DISTRIBUTION SYSTEM}

As noted by Standridge and Marvel (2006), lean concepts are applicable to supply chains and distribution systems as well as manufacturing systems. The case study described here involves a distribution system for parts used in assembling signs. This system is composed of approximately 100 active vendors which distribute approximately 5,000 stock keeping units (SKUs) to one distribution center (DC) and 19 branch warehouses located throughout the continental United States. The various SKUs are grouped into product lines. The DC followed a regular shipping schedule to each of the branch warehouses.

Customers of the system purchase parts on a daily basis at the branch warehouses and the DC (i.e., the DC also acted as a branch warehouse). The branch warehouses replenish their inventories by ordering stock either from a vendor or from the DC, while the DC always orders its stock directly from a vendor. The main decisions of interest had to do with whether the branch warehouses should order stock directly from a vendor or through the DC; these decisions could vary by product line and branch warehouse.

The branch warehouses use different ordering policies depending on whether they order from the DC or the vendor. When a branch warehouse ordered its stock for a product line from the DC, an (s, S) policy was followedthat is, when an inventory level reached the reorder point, $\mathrm{s}$, for any SKU in the product line, an order was placed to the DC for the 'order up to $S$ ' amount. The values of $s$ and $S$ could vary by SKU and were dependent on parameters such as forecast demand and lead time from the DC as determined from the DC's shipping schedule. 
When a branch warehouse orders stock for any SKU for a product line directly from a vendor, a "class method" is used in setting the ordering policy. With this method, whenever the inventory for a SKU falls below a particular reorder point, an order is placed with the vendor for a prescribed order quantity. In most cases, a vendor has a minimum order quantity required for a product line; in these situations, a branch warehouse would sometimes add SKUs to the order that had inventory levels above their respective reorder points to meet the vendor minimum-i.e., the branch warehouse in question would have to make a decision as to whether to add additional SKUs to an order, or let a particular SKU (or SKUs) fall even further below their reorder points. The values set for the reorder points and reorder quantities for a particular SKU depended upon the projected demand and lead time for that SKU from the vendor. A safety factor was used in many cases when the demand and/or the lead time were highly volatile. The demand at the DC for any particular SKU in a product line was dependent on which branch warehouses had to be supplied that product line by the DC.

A simulation model, using the Arena software package (Kelton, Sadowski, and Sturrock 2007) was built to represent the distribution system. Several different types of control variables can be input to the model; however, the main type investigated in this case study, as mentioned earlier, involved whether each branch orders a product line directly from the vendor, or through the DC. These are represented as zero-one variables, where $X_{j}=0$ means that branch $\mathrm{j}$ orders a product line directly from the vendor for that product line, and $\mathrm{X}_{\mathrm{j}}=1$ means that branch $\mathrm{j}$ orders the product line through the DC.

Various performance measure values are output by the model, including sales dollars, lost sales (which occur when there is a demand for a SKU and there is zero inventory available to supply that demand), shipping charges, inventory carrying charges, and cost of purchasing SKUs from the vendor. However, the two main performance measures of interest were lost sales (denoted as $\mathrm{X} 1$ ) and inventory carrying charges (denoted as X2) for the entire system.

Also, in order to keep this case study brief in nature, only one product line, consisting of 27 different SKUs, is considered. This gives us an optimization problem involving 19 zero-one decision variables; hence, considering all possible combinations of 19 zeros and ones, there are 2 to the $19^{\text {th }}$ power possible solutions to this problem.

Runs of the model were set up for a one-year duration, with a warm up period of 90 days. The current policy used by the distribution system (called the "Authorized Replenishment Plan") for this product line involved having all 19 branches ordering the product line through the DC, except for branches 5,8, and 13. Running this policy through the simulation model resulted in values of $\mathrm{X} 1=$ Lost Sales of $\$ 21,001$ and X2 = System Inventory
Carrying Charges of $\$ 85,776$ over the 275 days for which data was collected for the model.

The management of the distribution system was interested in achieving a leaner system - that is, one in which inventory levels were reduced - but not at the expense of increasing lost sales an inordinate amount. A simple criterion model was used in order to achieve a leaner system:

$$
\text { Minimize X1 = Lost Sales, }
$$

subject to X2 (System Inventory Carrying Charges) $\leq \mathrm{C}$, where $\mathrm{C}$ was to be determined.

The OptQuest optimization tool was used with the above criterion model, in order to choose the "best set of values" for the 19 zero-one control variables. Initially, C was set at a value of $\$ 80,000$, which led to an optimal solution which gave values for Lost Sales of \$11,144 and System Inventory Carrying Charges of $\$ 72,196$, an improvement of both performance measure values.

Following this initial optimization, the constraint on system inventory carrying charges was tightened, by setting $C$ at respective values of $\$ 60,000, \$ 50,000, \$ 40,000$, and $\$ 30,000$; solving these optimization problems led to the following sets of performance measure values:

1. Lost Sales $=\$ 11,694$, Inv. $\mathrm{CC}=\$ 57,726$.

2. Lost Sales $=\$ 18,973$, Inv. $\mathrm{CC}=\$ 49,727$.

3. Lost Sales $=\$ 33,689$, Inv. $\mathrm{CC}=\$ 38,745$.

4. Lost Sales $=\$ 93,156$, Inv. $\mathrm{CC}=\$ 28,644$.

Outcomes 3 and 4 above were viewed as unacceptable in terms of lost sales, while either the first or second outcomes were viewed as acceptable. Each of these policies allowed for a leaner system in terms of inventory carrying charges, but not at the expense of increased lost sales.

\section{SUMMARY}

Lean principles require the identification of current and ideal states and the identification of the policy that will lead to an ideal state. An ideal state can be defined in terms of values for various performance measures associated with a system. Since typically, tradeoffs need to be made between various performance measures, an ideal state as initially defined may not be attainable.

Using principles as procedures from the area of multi-objective decision analysis, an appropriate criterion model can be identified which, when used with an optimization procedure and simulation model, will allow for the identification of an ideal system state along with a policy that will lead to that ideal state.

\section{ACKNOWLEDGEMENTS}

This research was partially funded by the NSF sponsored Center for Engineering Logistics and Distribution at the University of Louisville. 


\section{REFERENCES}

Anderson, N. P., G. W. Evans, and W. E. Biles. 2006. Simulation optimization of logistics systems through the use of variance reduction techniques and criterion models. Engineering Optimization 3:441-460.

April, J., M. Better, F. Glover, and J. Kelley. 2004. New advances and applications for marrying simulation and optimization. In Proceedings of the 2004 Winter Simulation Conference, ed. R. G. Ingalls, M. D. Rosetti, J. S. Smith, and B. A. Peters, 80-86. Piscataway, New Jersey: Institute of Electrical and Electronics Engineers, Inc. .

Butler, J., D. J .Morrice, and P. W. Mullarkey. 2001. A multiple attribute utility theory approach to ranking and selection. Management Science 47:800-816.

Cambron, K. and G. W. Evans. 1991. Use of the analytic hierarchy process for multiobjective facility layout. Computers and Industrial Engineering 20:211-229.

Clemen, R. T. 1996. Making Hard Decisions: An Introduction to Decision Analysis. 2nd ed. Pacific Grove, CA: Duxbury Press.

Evans, G.W., W.E. Biles, and S.M. Alexander. 1992. A large-scale simulation model for analyzing the production of pipe valves and fittings. Simulation 59: 366-374.

Evans, G.W. and R. Fairbairn. 1989. Selection and scheduling of advanced missions for NASA using 0-1 integer linear programming. Journal of the Operational Research Society 40:971-982.

Jain, S. 2004. Supply chain management tradeoffs analysis. In Proceedings of the 2004 Winter Simulation Conference, ed. R. G. Ingalls, M. D. Rosetti, J. S. Smith, and B. A. Peters, 1358-1364. Piscataway, New Jersey: Institute of Electrical and Electronics Engineers, Inc. .

Keeney, R. L. 1992. Value Focused Thinking. Harvard University Press, Cambridge, MA.

Keeney, R. L. and H. Raiffa. 1993. Decisions with Multiple Objectives: Preferences and Value Tradeoffs. 2nd ed. Cambridge University Press.

Kelton, W. D., R. P. Sadowski, and D.T. Sturrock. 2007. Simulation with Arena. 4th ed. McGraw Hill.

Kharwat, A. K. 1991. Computer simulation: an important tool in the fast-food industry. In Proceedings of the 1991 Winter Simulation Conference, 811. Piscataway, New Jersey: Institute of Electrical and Electronics Engineers, Inc.

Lee, L.H., E. P. Chew, S. Teng, and D. Goldsman. 2004. Optimal computing budget allocation for multiobjective simulation models. In Proceedings of the 2004 Winter Simulation Conference, ed. R.G. Ingalls, M. D. Rosetti, J. S. Smith, and B. A. Peters, 586-594. Piscataway, New Jersey: Institute of Electrical and Electronics Engineers, Inc. .
Love Jr., R. R. and J. M. Hoey. 1990. Management science improves fast-food operations. Interfaces 20:2129.

Manheim, M. L. and F. Hall. 1967. Abstract representtation of goals: a method for making decisions in complex problems. In Transportation: A Service, Proceedings of the Sesquicentennial Forum. New York: New York Academy of Sciences-American Academy of Mechanical Engineers.

Schwetman, H. 2000. Optimizing Simulations with CSIM18/OptQuest: Finding the Best Configuration. In Proceedings of the 2000 Winter Simulation Conference, 268-273. Piscataway, New Jersey: Institute of Electrical and Electronics Engineers, Inc. .

Standridge, C. R. and J. H. Marvel. 2006. Why lean needs simulation. In Proceedings of the 2006 Winter Simulation Conference, ed. L. F. Perrone, F. P. Wieland, J. Liu, B. G. Lawson, D.M. Nicol, and R. M. Fujimoto, 1907-1913. Piscataway, New Jersey: Institute of Electrical and Electronics Engineers, Inc. .

\section{AUTHOR BIOGRAPHIES}

GERALD W. EVANS is a Professor in the Department of Industrial Engineering at the University of Louisville. He has a B.S. degree in Mathematics, and M.S. and Ph.D. degrees in Industrial Engineering, all from Purdue University. Before entering academia, he worked as an Industrial Engineer for Rock Island Arsenal, and as a Senior Research Engineer for General Motors Research Laboratories. Besides simulation modeling and analysis, his research interests include multi-objective optimization, decision analysis, and discrete optimization. He can be reached by e-mail at <gwevanolelouisville.edu $>$.

SURAJ M. ALEXANDER is a Professor in the Department of Industrial Engineering at the University of Louisville. He has a B.S. degree in M.E. from I.I.T, Madras, and M.S. and Ph.D. degrees in Industrial Engineering and Operations Research, from the Virginia Polytechnic Institute and State University. Before entering academia, he worked as an Equipment Design Engineer for Corning, Inc. His current research interests are in the areas of quality assurance, process quality monitoring, diagnosis and control, lean engineering, and logistics. Dr. Alexander is a Fellow of the Institute of Industrial Engineers and the American Society of Quality. He is a Registered Professional Engineer in Kentucky, and a Certified Quality Engineer. He can be reached by e-mail at $<\mathrm{su}-$ raj.alexander@louisville.edu>. 